\title{
Comparison of coprological, immunological and molecular methods for the detection of dogs infected with Angiostrongylus vasorum before and after anthelmintic treatment
}

\author{
M. $\mathrm{SCHNYDER}^{1} *$ R. JEFFERIES ${ }^{2,3} \dagger, \mathrm{A} \cdot \mathrm{SCHUCAN}^{1}, \mathrm{E} \cdot \mathrm{R} \cdot \mathrm{MORGAN}^{2,3} \dagger$ and \\ P. DEPLAZES ${ }^{1} \dagger$ \\ ${ }^{1}$ Institute of Parasitology, University of Zurich, Winterthurerstrasse 266a, 8057 Zurich, Switzerland \\ ${ }^{2}$ School of Veterinary Science, University of Bristol, Langford, BS40 5DU, UK \\ ${ }^{3}$ School of Biological Sciences, University of Bristol, Woodland Road, Bristol, BS8 1UG, UK
}

(Received 24 Fanuary 2015; revised 3 April 2015; accepted 25 April 2015; first published online 3 Fune 2015)

\section{S UMMARY}

Timely diagnosis of the nematode Angiostrongylus vasorum in dogs is important in view of severe and permanent lung and cardiovascular lesions that may occur. The performance of the classical Baermann coprological method was compared with ELISAs for the serological detection of circulating antigen and specific antibodies and with Polymerase chain reaction (PCR) performed on EDTA blood, feces and tracheal swabs of serial samples from experimentally inoculated dogs over 13 weeks post inoculation (wpi) $(n=16)$ and following anthelmintic treatment $(n=6)$. Patency was observed from 6.7 to $7.6 \mathrm{wpi}$ in all dogs, Baermann results were then mostly positive $(116 / 119,97 \%)$ during the patent period, with wide variations in the numbers of first stage larvae numbers. Blood PCR was tested positive on 1-2 occasions in 11/16 dogs in the pre-patent period, while all tested positive by antibody-detection ELISA by 6 wpi. The proportion of dogs testing positive by fecal PCR and antigen-detection ELISA rose early in the patent period. Tracheal swabs were occasionally DNA-positive in 3/16 dogs starting from 10 wpi. Following treatment, larval excretion stopped within 3 weeks and blood PCR results became negative within 1 week $(5 / 6 \mathrm{dogs})$, while $4 / 6$ dogs were positive for parasite DNA in tracheal swabs. Parasite antigen and specific antibodies both persisted in the blood for 3-9 weeks after treatment, with average optical densities and the proportion of positive dogs falling gradually, while results using other tests were much more variable. Results indicate that the earliest and most consistent results are obtained by the ELISAs, which can also be used for monitoring dogs after anthelmintic treatment.

Key words: Angiostrongylus vasorum, dog, copromicroscopy, serology, antigen detection, antibody detection, PCR, tracheal swabs, fecal samples, blood.

\section{INTRODUCTION}

Angiostrongylus vasorum is a metastrongylid nematode residing in the right heart and pulmonary arteries of its definitive canid host (Guilhon, 1963). It is a cause of respiratory distress and diverse other clinical signs (Koch and Willesen, 2009), and can be severe or even fatal in dogs (Cury and Lima, 1996; Staebler et al. 2005). The recent increase in reports of canine angiostrongylosis throughout Europe (Morgan and Shaw, 2010) and North America (Conboy, 2011; Kistler et al. 2014) suggests spread and/or increased awareness of this emerging parasitic disease. Accordingly, novel highly sensitive and specific diagnostic methods have been developed, to complement the currently used larval migration techniques such as the Baermann-Wetzel technique

\footnotetext{
* Corresponding author. Vetsuisse Faculty, Institute of Parasitology, University of Zurich, Winterthurerstrasse 266a, 8057 Zürich, Switzerland. E-mail: manuela.schnyder@uzh.ch

$\dagger$ These authors contributed equally to this paper.
}

(Deplazes et al. 2013) for the detection of first stage larvae (L1) in fecal samples. While simple fecal smears may be used for fast diagnosis in general practice in strongly suspected cases (Humm and Adamantos, 2010), and fecal flotation methods such as FLOTAC can also work reasonably well (Schnyder et al. 2010b), copromicroscopic methods in general suffer from the problem of reduced sensitivity due to low or intermittent larval shedding (Oliveira-Junior et al. 2006) especially in cases of low worm burdens. The sensitivity of larval detection is also restricted when analysing posted fecal samples that arrive with some delay at the laboratory and therefore containing deformed or dead larvae. Also, pre-patent infections are not detected. Furthermore, since other lung-worm larvae such as Crenosoma vulpis and Filaroides spp. (McGarry and Morgan, 2009; Deplazes et al. 2013) occur in dog feces, specificity may depend on the expertise of diagnostic staff.

Recently developed serological methods (Schnyder et al. 2011; Schucan et al. 2012) for the detection of 
A. vasorum infected dogs by ELISAs (Enzyme-linked Immunosorbent Assays) represent a valid alternative for diagnosis in individuals and have been evaluated and used in the field in large scale epidemiological surveys (Guardone et al. 2013; Schnyder et al. $2013 a, b)$.

A further alternative for the diagnosis of $A$. vasorum infected dogs is provided by molecular tools such as Polymerase chain reaction (PCR). In particular, a PCR procedure developed to facilitate the processing of larger fecal volumes was described as a non-invasive tool for wildlife surveillance in foxes (Al-Sabi et al. 2010) and may be recommended for prevalence studies in intermediate mollusc hosts (Ferdushy et al. 2009). Recently developed real-time PCR assays were able to detect blood, feces or intermediate host tissue spiked with a single $A$. vasorum L1 with high specificity. Discordance between blood and fecal PCR (and Baermann) results in naturally infected dogs was suggested to be due to the intermittent presence of circulating DNA (Jefferies et al. 2009). The authors suggested that accurate detection limits would be obtained through the use of samples from experimentally infected animals after known inoculation times.

The aim of this study was to compare different diagnostic methods for $A$. vasorum after experimental infection, using blood, fecal and tracheal swab samples. By taking serial samples, tests are compared in relation to suitability at different stages of clinical infection, as well as providing possible insights into the dynamics of the infection process.

\section{MATERIALS AND METHODS}

\section{Dog sera, tracheal swabs, fecal samples}

A total of 16 dogs were experimentally inoculated with 200 third stage larvae (L3) of $A$. vasorum harvested from experimentally infected Biomphalaria glabrata snails as previously described (Schnyder et al. 2009). Six dogs were treated with imidacloprid $10 \mathrm{mg} \mathrm{kg}^{-1}$ body weight (BW)/moxidectin $2 \cdot 5 \mathrm{mg} \mathrm{kg}^{-1}$ BW spot-on (Advocate ${ }^{\circledR}$, Bayer Animal Health) 13 weeks ( 88 days) post inoculation (wpi). Ethical clearance for the study was obtained from the Cantonal Veterinary Office of Zurich (permission number 185/2008); the dogs were facility-born animals and the study was primarily conducted to investigate drug efficacy for parasite clearance, with the present data supporting that aim and utilizing the same filed permissions. Due to further research purposes (i.e. cardiology studies - see Matos et al. 2012 - and production of excretory/secretory antigens), $10 \mathrm{dogs}$ were left untreated and 9 of them were necropsied between 11 and 51 wpi. At necropsy, the worm burden was determined through dissection and retrograde lung perfusion, as previously described (Schnyder et al. 2009).
Peripheral blood was regularly (mostly every 1-2 weeks) collected from the jugular vein into BD Vacutainer $^{\circledR}$ tubes (Becton Dickinson, Franklin Lakes, NJ, USA) for collection of EDTA (Ethylenediaminetetraacetic acid) anticoagulated samples (for PCR) and serum samples (for ELISAs). Fecal samples and tracheal swabs were collected contemporaneously. The number of L1 per gram of feces (LPG) was determined on each occasion by the BaermannWetzel technique (Deplazes et al. 2013) on $10 \mathrm{~g}$ of fresh feces, with remaining feces, blood, serum and swabs frozen at $-20^{\circ} \mathrm{C}$ pending processing.

\section{ELISAs for the detection of $A$. vasorum circulating antigen and specific antiparasitic antibodies}

A sandwich-ELISA based on monoclonal and polyclonal antibodies directed against $A$. vasorum adult excretory/secretory (E/S) - antigen and having a sensitivity of $95 \cdot 7 \%$ and specificity of $94.0 \%$ was performed for detection of circulating antigen as described in Schnyder et al. (2011). For detection of specific antibodies an ELISA using $A$. vasorum adult E/S antigen with a sensitivity of $85.7 \%$ and a specificity of $98 \cdot 8 \%$ (Schucan et al. 2012) was used. Absorbance values were read at $405 \mathrm{~nm}$ with a Multiscan RC ELISA reader (Thermo Labsystems, Helsinki, Finland). All test runs included a background control, a conjugate control, three positive control sera from three experimentally infected dogs and two negative control sera from uninfected dogs. The cut-off value of each test was determined as described: for the antigen detection ELISA, the cut-off was determined using the mean value of optical density (OD, $\mathrm{A}_{405 \mathrm{~nm}}$ ) plus 3 standard deviations (S.D.) of 30 sera from randomly chosen samples submitted to the Veterinary Laboratory of the Veterinary Hospital of the Vetsuisse Faculty of the University of Zurich for various reasons not related to suspected parasitological infections (cut-off $\mathrm{OD}=0 \cdot 159)$. For the antibody detection ELISA, 30 sera from healthy blood donor dogs, which were subjected to intense and regular clinical and laboratory screening analyses, and not originating from a known $A$. vasorum endemic area in Switzerland, were used to determine the cut-off value. Four S.D. were applied in this case for better discrimination between positive and negative sera, based on the results with predefined sera (cut-off OD $=0 \cdot 310$ )

\section{PCR}

DNA extraction and real-time PCR were performed according to the method described by Jefferies $e t$ al. (2009). Briefly, DNA was extracted using a DNEasy blood and tissue kit (Qiagen, Germany, for whole blood and tracheal swabs) and QIAamp stool kit (Qiagen, Germany, for feces) according the manufacturers' instructions. Specific primers designed to 
amplify a region of the second ribosomal transcribed spacer (ITS-2) of $A$. vasorum were used in a realtime PCR with defined procedures and cut-off limits. This assay has previously been shown to be highly specific for $A$. vasorum and to detect a single molecule of plasmid DNA or a single L1 (Jefferies et al. 2009).

\section{RESULTS}

Patency (L1 detected in feces) was observed starting from $6 \cdot 7$ to $7 \cdot 6$ wpi in all dogs, and larval excretion in dogs treated at 13 wpi stopped within 3 weeks after treatment. The different tests detected infection at different stages, and varied in the internal (within-dog over time) and external (between-dog) consistency of results. Thus, an example profile from an individual $\mathrm{dog}$ is presented in Table 1 . Infection in this case was first detected by blood PCR approximately 2 weeks after infection, but blood PCR results were inconsistently positive after this time up until treatment and 5 weeks thereafter, when the test became consistently negative. Antibodies against $A$. vasorum were also detected during the pre-patent period, from 4 weeks after infection, and were consistently present at abovethreshold levels until 5 weeks after treatment, when OD dropped below the threshold for a positive result. Circulating antigens in serum, and L1 and parasite DNA in the feces were observed from around 7 wpi, and declined over 2-4 wpi.

All dogs were found to shed L1 by 8 weeks after inoculation, by which time variable proportions of other tests were positive (Fig. 1, Table 2). Baermann results were then mostly positive during the patent period, though occasional negative tests were observed and the LPG varied widely between and within dogs. Antibody-detection ELISA and blood PCR tested positive in some dogs in the pre-patent period, with the proportion of dogs testing positive by larval migration, fecal PCR and antigen-detection ELISA rising early in the patent period. All the infected dogs were tested positive by antibody-detection ELISA by 6 wpi and remained consistently so until after treatment (or the end of the experiment in untreated dogs that remained infected). All dogs also were antigen-positive at $11 \mathrm{wpi}$ and remained so until after treatment (or the end of the experiment in untreated dogs that remained infected). In contrast, results using other tests were much more variable ('Table 2). Following treatment at $13 \mathrm{wpi}(n=6$ dogs), blood PCR results became negative within 1 week, with one positive result observed in a single $\operatorname{dog}(1 / 6) 4$ weeks after treatment. The proportion of dogs excreting L1 declined over the 3 weeks following treatment. In contrast, dogs testing positive for parasite DNA in tracheal swabs increased from $3 / 16$ dogs being occasionally positive before $12 \mathrm{wpi}$ to $4 / 6$ dogs being positive after treatment until 3 weeks later (15 wpi), while also 4 out of 10 untreated dogs were irregularly positive until 15 wpi. Parasite antigen and specific antibodies both persisted in the blood up to 7 and 9 weeks, respectively, after treatment (Table 2), with average ODs and the proportion of positive dogs falling gradually in the 5 weeks after treatment over which monitoring continued for all $6 \mathrm{dogs}$ (Fig. 1). Three untreated dogs spontaneously ceased to excrete L1 during the experiment, in weeks $15(n=2)$ and $17(n=1)$ after inoculation, and no worms were found on post-mortem examination following euthanasia $(n=2,9$ days after first negative Baermann's result). In these dogs, blood PCR rapidly became negative after cessation of larval shedding, while antibody and antigen ELISAs were still positive. Three of the 6 treated dogs were euthanized at the end of the experiment, and no worms were recovered after dissection.

The number of L1 recovered by Baermann examination varied widely between dogs, and fluctuated over time within individual dogs. Thus, although all dogs were inoculated with 200 L3, LPG during the patent period varied from 0 to $2010 \mathrm{~g}^{-1}$ (mean 122, s.D. 362). Average LPG over the patent period varied between dogs from $0 \cdot 25$ to 456 . The number of worms recovered from untreated dogs that retained their infections and were subjected to postmortem worm counts $(n=7)$ also varied (mean 35 , range $13-68$, S.D. 17), but the coefficient of variation (CV) $(0.49, n=7)$ was inferior to that of average larval count over the patent period between dogs $(1 \cdot 69, n=16)$ as well as to that of weekly larval count in individual dogs over the patent period (range $0 \cdot 54-2 \cdot 07$, median $1 \cdot 28, n=16$ ). Results of other tests were less variable within dogs over time than was LPG (median CV and range: for blood PCR Ct (Cycle threshold) $=0.033 ; 0.003-0.066$, for fecal PCR $\mathrm{Ct}=0 \cdot 16 ; 0 \cdot 14-0 \cdot 20, \mathrm{Ag}$ ELISA $\mathrm{OD}=0 \cdot 55 ; \quad 0 \cdot 22-0 \cdot 75, \quad \mathrm{Ab}$ ELISA $\mathrm{OD}=0 \cdot 10$; $0 \cdot 09-0 \cdot 17)$. There was no correlation between any test result when averaged over the patent period and the post-mortem worm count in the same individual $(n=7)$.

\section{DISCUSSION}

Early diagnosis of infection with $A$. vasorum could be important in clinical case management, since pre-patent lung pathology and clinical disease can occur (Schnyder et al. 2009). The present results indicate that circulating specific antibodies and parasite DNA in blood can be detected during the prepatent period. The earliest indicator of infection was provided in a single dog by blood PCR 2 wpi, but this was not a consistent finding, with all four positive blood PCR tests in the first 5 weeks of infection being followed by negative results in the same dogs. It is likely that these early positive PCR results relate to DNA originating from L3 during 
Table 1. Example time-course of test results from a selected individual dog during infection with Angiostrongylus vasorum before (a) and after (b) anthelmintic treatment 13 weeks after inoculation. Inoculation with infectious third stage larvae occurred on day zero. ' + ' = positive results, ' - ' = negative results, blank cells $=$ the test was not performed. Data are illustrative of individual level information that was subsumed into the group-level summaries presented elsewhere.

\begin{tabular}{|c|c|c|c|c|c|c|c|c|c|c|c|c|c|}
\hline \multicolumn{14}{|l|}{ (a) (Prior to treatment) } \\
\hline $\begin{array}{l}\text { Day after inoculation } \\
(\text { week })^{\mathrm{a}}\end{array}$ & $-7(-1)$ & $6(1)$ & $13(2)$ & $20(3)$ & $27(4)$ & $\begin{array}{l}34 / 35 \\
(5)\end{array}$ & $\begin{array}{l}41 / 42 / 43 \\
(6)\end{array}$ & $48 / 49(7)$ & $\begin{array}{l}55 / 56 / 57 \\
(8)\end{array}$ & $\begin{array}{l}62 / 63 / 64 \\
(9)\end{array}$ & $\begin{array}{l}69 / 70 \\
(10)\end{array}$ & $\begin{array}{l}75 / 76 \\
(11)\end{array}$ & $\begin{array}{l}83 / 84 \\
(12)^{\mathrm{b}}\end{array}$ \\
\hline Blood PCR $\left(\mathrm{C}_{\mathrm{t}}\right.$ values ${ }^{\mathrm{c}}$ & & & $+(36 \cdot 84)$ & -0 & -0 & -0 & -0 & $+(35 \cdot 81)$ & -0 & $+41 \cdot 71$ & -0 & -0 & $+35 \cdot 50$ \\
\hline Fecal PCR $\left(\mathrm{C}_{\mathrm{t}} \text { values }\right)^{\mathrm{c}}$ & & & & & & & & & $+40 \cdot 52$ & & & $+34 \cdot 53$ & $+40 \cdot 69$ \\
\hline Swab PCR $\left(\mathrm{C}_{\mathrm{t}} \text { values }\right)^{\mathrm{c}}$ & & & & & & -0 & -0 & -0 & -0 & -0 & -0 & $+39 \cdot 01$ & \\
\hline Baermann $(L P G)^{\mathrm{d}}$ & -0 & -0 & -0 & -0 & -0 & -0 & -0 & $+0 \cdot 1$ & $+1 \cdot 0$ & $+1 \cdot 1$ & $+11 \cdot 2$ & $+14 \cdot 4$ & $+59 \cdot 2$ \\
\hline AG ELISA (OD-value) ${ }^{\mathrm{e}}$ & $-0 \cdot 057$ & $-0 \cdot 067$ & $-0 \cdot 066$ & $-0 \cdot 052$ & $-0 \cdot 066$ & $-0 \cdot 065$ & $-0 \cdot 051$ & $-0 \cdot 063$ & $+0 \cdot 177$ & +0.953 & $+1 \cdot 439$ & $+1 \cdot 610$ & $+1 \cdot 633$ \\
\hline AB ELISA (OD-value) ${ }^{\mathrm{f}}$ & $-0 \cdot 005$ & $-0 \cdot 018$ & $-0 \cdot 125$ & $-0 \cdot 289$ & $+0 \cdot 433$ & $+0 \cdot 927$ & $+1 \cdot 272$ & $+1 \cdot 252$ & $+1 \cdot 138$ & $+0 \cdot 935$ & $+0 \cdot 650$ & $+0 \cdot 873$ & $+0 \cdot 725$ \\
\hline \multicolumn{14}{|l|}{ (b) After treatment } \\
\hline \multicolumn{3}{|c|}{ Day after inoculation (week after treatment) ${ }^{\mathrm{a}}$} & $97(2)$ & \multicolumn{2}{|c|}{$104(3)$} & $111(4)$ & $118(5)$ & $132 / 133(7)$ & $146(9)$ & $160(11)$ & $174(13)$ & $188(15)$ & $230(21)$ \\
\hline \multicolumn{3}{|l|}{ Blood PCR $\left(\mathrm{C}_{\mathrm{t}} \text { values }\right)^{\mathrm{c}}$} & -0 & \multicolumn{2}{|l|}{-0} & -0 & $+40 \cdot 58$ & -0 & \multicolumn{2}{|l|}{-0} & \multicolumn{2}{|l|}{-0} & \multirow{3}{*}{$\begin{array}{l}-0 \\
-0\end{array}$} \\
\hline & $+35 \cdot 51$ & \multirow{2}{*}{\multicolumn{2}{|c|}{-0}} & -0 & -0 & -0 & -0 & -0 & \multirow[t]{2}{*}{-0} & \multirow[t]{2}{*}{-0} & \\
\hline & \multicolumn{2}{|c|}{ Swab PCR $\left(C_{t} \text { values }\right)^{c}$} & -0 & & & & & & & & & & \\
\hline Baermann $(L P G)^{\mathrm{d}}$ & & & $+291 \cdot 6$ & \multicolumn{2}{|c|}{$+0 \cdot 1$} & -0 & -0 & -0 & -0 & -0 & \multirow{3}{*}{$\begin{array}{l}-0 \\
-0 \cdot 056\end{array}$} & \multirow[t]{3}{*}{-0} & \multirow{3}{*}{$\begin{array}{l}-0 \\
-0 \cdot 063\end{array}$} \\
\hline AG ELISA (OD-values) & & & $+1 \cdot 575$ & \multirow{2}{*}{\multicolumn{2}{|c|}{$\begin{array}{l}+1 \cdot 370 \\
+0 \cdot 493\end{array}$}} & $+0 \cdot 707$ & $-0 \cdot 068$ & $-0 \cdot 062$ & \multirow{2}{*}{\multicolumn{2}{|c|}{$\begin{array}{l}-0 \cdot 056 \\
-0 \cdot 139\end{array}$}} & & & \\
\hline AB ELISA (OD-values) ${ }^{f}$ & & & $+0 \cdot 604$ & & & $+0 \cdot 425$ & $+0 \cdot 348$ & $-0 \cdot 183$ & & & & & \\
\hline
\end{tabular}

PCR, polymerase chain reaction; LPG, larval density per gram of feces; OD, optical density

a Sampling for different tests sometimes occurred over consecutive days, and week markers are approximate.

b Treatment with Advocate ${ }^{\circledR}$ (Bayer Animal Health, imidacloprid $10 \mathrm{mg} \mathrm{kg}^{-1}$ body weight (BW)/moxidectin $2.5 \mathrm{mg} \mathrm{kg}{ }^{-1}$ BW spot-on) 88 days after inoculation.

c Cycle threshold of the quantitative real-time PCR (Jefferies et al. 2009).

d Number of larvae per gram of feces obtained by Baermann-Wetzel examination (Deplazes et al. 2013).

e Detection of circulating antigen by ELISA (Schnyder et al. 2011), with optical density absorbance values read at $405 \mathrm{~nm}$, cut-off value $=0 \cdot 159$.

${ }^{\mathrm{f}}$ Detection of specific antibodies by ELISA (Schucan et al. 2012), with optical density absorbance values read at $405 \mathrm{~nm}$, cut-off value $=0 \cdot 310$. 


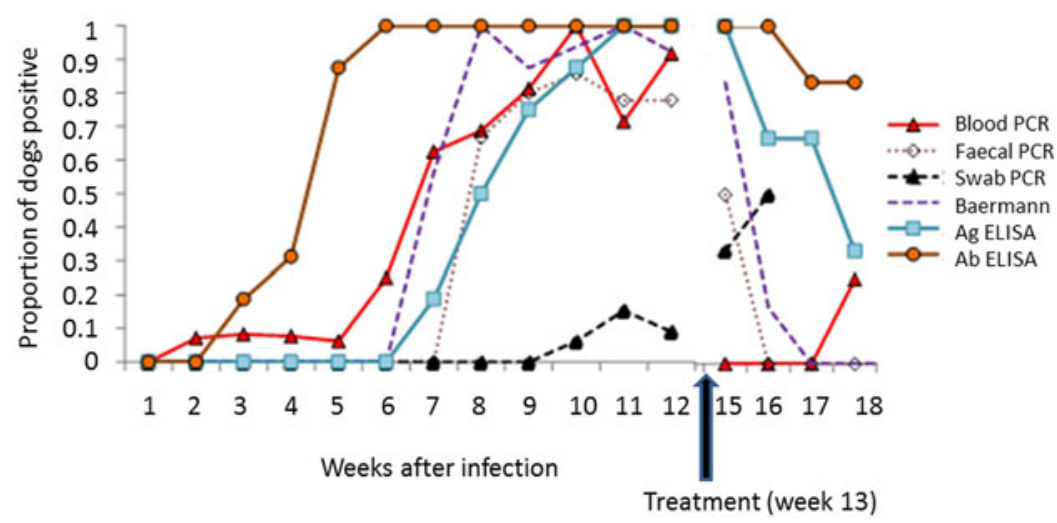

Fig. 1. The detection of Angiostrongylus vasorum in experimentally infected dogs using different methods during the early phase of infection $(n=16)$, and after anthelmintic treatment $\left(n=6\right.$; arrow) with imidacloprid-moxidectin $\left(\right.$ Advocate $\left.{ }^{\circledR}\right)$ in week 13 .

Table 2. Consistency of test results in detecting infection in dogs experimentally inoculated with Angiostrongylus vasorum ( $n=16$ inoculated; of which $n=6$ treated with the anthelmintic imidacloprid $10 \mathrm{mg}$ $\mathrm{kg}^{-1}$ body weight (BW)/moxidectin $2 \cdot 5 \mathrm{mg} \mathrm{kg}^{-1} \mathrm{BW}$ spot-on (Advocate ${ }^{\circledR}$, Bayer Animal Health) at 13 weeks after inoculation.

\begin{tabular}{|c|c|c|c|c|c|}
\hline Test ${ }^{\mathrm{a}}$ & $\begin{array}{l}\text { First positive, } \\
\text { weeks after } \\
\text { inoculation } \\
\text { mean (range) }\end{array}$ & $\begin{array}{l}\text { Proportion } \\
\text { positive, } \\
\text { within dogs } \\
\text { mean (range) }\end{array}$ & $\begin{array}{l}\text { Total } \\
\text { tests }\end{array}$ & $\begin{array}{l}\text { Total } \\
\text { proportion } \\
\text { positive }\end{array}$ & $\begin{array}{l}\text { Last positive, } \\
\text { weeks after } \\
\text { treatment } \\
\text { mean }\left(\text { range) }{ }^{\mathrm{f}}\right.\end{array}$ \\
\hline Blood PCR & $6 \cdot 3(2-10)$ & $0 \cdot 78(0 \cdot 45-1)$ & 120 & $0 \cdot 70$ & $1 \cdot 0(1-5)$ \\
\hline Fecal PCR & $9 \cdot 1(8-11)$ & $0 \cdot 91(0 \cdot 5-1)$ & 58 & $0 \cdot 84$ & $2 \cdot 0(-)$ \\
\hline Swab PCR & $12 \cdot 9(10-15)$ & $0 \cdot 71(0 \cdot 5-1)$ & 16 & $0 \cdot 69$ & $2 \cdot 7(2-3)$ \\
\hline Baermann & $7 \cdot 4(7-8)$ & $0.95(0.4-1)$ & 119 & 0.97 & $2 \cdot 2(2-3)$ \\
\hline AG ELISA & $8 \cdot 7(7-11)$ & $1(-)$ & 97 & 1 & $4 \cdot 0(3-7)$ \\
\hline AB ELISA & $4 \cdot 6(3-6)$ & $1(-)$ & 158 & 1 & $5 \cdot 7(3-9)$ \\
\hline
\end{tabular}

$\mathrm{PCR}$, polymerase chain reaction

a For test details see Table 1 .

b Week after infection in which each test returned a positive result, on average (range in brackets).

c Average proportion of each repeated test that was positive within individuals, after the first positive and until treatment, euthanasia or spontaneous clearance of worms (range in brackets).

$\mathrm{d}$ Total number of each test that was run after the first positive.

e Proportion of all dogs that tested positive of the total number of tests that was run after the first positive.

$\mathrm{f}$ The average number of weeks after treatment that individual dogs still tested positive (range in brackets).

migration and maturation, in which case inconsistent presence in circulating blood is not surprising. Specific anti- $A$. vasorum antibodies, on the other hand, were detected at above-threshold levels from week 3 in some dogs, and from week 5 in all dogs, and once positive remained positive throughout the patent period. Ab-detection ELISA is therefore the most promising test for identifying dogs at an early stage of infection.

Although diagnosis early in the course of infection might confer clinical advantages in some cases, natural infections are likely to present clinically at a wide range of intervals following infection. The most useful diagnostic tests to support infection are therefore those that are most consistently positive in genuinely infected dogs. This is especially so because in canine angiostrongylosis, the clinical consequences of failing to diagnose an infection are likely to be higher than those of unnecessarily treating an uninfected dog (Koch and Willesen, 2009; Morgan and Shaw, 2010; Paradies et al. 2013). The present study identified very high variation in test results that relied on the presence of parasite material in the feces (Baermann-Wetzel examination and fecal PCR) or sputum (tracheal swab PCR), or parasite DNA in the blood (blood PCR). In contrast, antibody and antigen detection ELISAs were consistently positive in all dogs throughout the patent period, and confirmed to be useful in a clinical setting. These results also confirm previous studies showing that the antigen detection ELISA was able to detect circulating antigen already starting 
from 5 weeks after experimental inoculation of dogs (Schnyder et al. 2011) and the specific antibody ELISA starting 2-3 weeks after inoculation (Schucan et al. 2012).

When using antibody detection as a clinical diagnostic test in an at-risk population, the persistency of antibodies in recently treated dogs needs to be considered. In the present study, dogs remained positive for antibodies for 3-9 weeks after treatment, more than for any other test. However, all tests remained positive in some dogs for a number of weeks after treatment (e.g. 5 weeks for circulating antigen and 4 weeks for blood PCR). The fecal tests showed the fastest decline in number of positives after treatment ( 1 and 2 weeks for fecal PCR and Baermann-Wetzel examination, respectively), but the high variation in results from those tests during patency weaken their interpretation as a test for successful treatment, unless multiple examinations are performed. Monitoring of dogs after treatment for successful parasite clearance could therefore make use of any of the tests evaluated, but perhaps most usefully would track the disappearance of circulating antigen and/or antibodies after the appropriate time after treatment.

PCR was described to screen throat swabs for Aelurostrongylus abstrusus in cats (Traversa et al. 2008), and a similar procedure for dogs infected with $A$. vasorum was considered. In this study, the number of dogs testing positive for $A$. vasorum DNA in sputum paradoxically increased after anthelmintic treatment. This could be explained by the presence of genetic material from dead and degrading worms, especially larvae, being carried up the bronchial muco-ciliary escalator. However, an increase in the number of positive tests occurred after 12 weeks in untreated as well as treated dogs, for which there is no obvious explanation. The late onset and highly inconsistent detection of DNApositive tracheal swabs suggest that this test would be of little use in diagnosing clinically affected dogs in practice, although PCR could be useful to confirm the identity of larvae discovered on airway cytology. Detection of DNA could also be enhanced by taking larger, well-mixed sputum samples, though the ease of sampling is likely to vary between individual dogs and clinical presentations.

The timing of serial test results in the present study appears to confirm existing understanding of the parasite life cycle and infection process. Thus, occasional and transient positive blood PCR results could indicate migrating larvae, with this process also triggering a humoral immune response and gradually increasing production of specific antibodies. The other tests (with exception of PCR with tracheal swabs) became positive in most dogs around week 7 , coinciding with establishment of adult parasites and production of eggs and L1, accompanied by release of parasite antigen and DNA into the circulation and trachea. The presence of circulating DNA of $A$. vasorum is additionally sustained by the occurrence of ectopic localization of the parasite in different organs and tissues, as reported for adult worms (King et al. 1994; Cury and Lima, 1996; OliveiraJunior et al. 2004) and L1 (Oliveira-Junior et al. 2004). The appearance of L1 in the feces 7-8 wpi is consistent with existing data on the pre-patent period in this species (Guilhon, 1963; Schnyder et al. $2010 a$ ). The fast disappearance of parasite DNA from circulating blood after anthelmintic treatment seems to suggest that PCR mainly detects DNA that is shed from living worms, e.g. during egg ejection or while producing $\mathrm{E} / \mathrm{S}$ antigen, rather than shed passively from worms in the blood vessels, in which case one might expect positive results for some time after treatment, as cells are lost from degrading worms. The longer persistence of detectable parasite antigen after treatment is consistent with such passive shedding into the blood. Interestingly, $3 / 10$ untreated dogs spontaneously lost their worm burden, accompanied by a decline in positive test results similar to that following treatment. The mechanisms involved in parasite clearance, and their relevance to natural infection and disease, are not known.

In total, $97 \%$ of fecal samples during the patent period yielded detectable L1, in contrast to previous reports of intermittent shedding of L1 in experimentally infected dogs (Oliveira-Junior et al. 2006). The average number of larvae recovered varied very widely between dogs, much more so than the number of adult worms recovered from those dogs subjected to post-mortem examination. Moreover, LPG fluctuated considerably over the course of infection in individual dogs. Given the additional inaccuracies conferred in practice by less sensitive fecal tests, threats to larval viability during transit, and variable operator experience, this could limit the sensitivity of diagnostic tests based on larval enumeration. Nevertheless, based on the here presented results, larval isolation through the Baermann method can still be recommended in combination with multiple fecal examinations by collection of feces over 3 consecutive days, as reported from recent prevalence studies performed with the Baermann funnel technique (Barutzki and Schaper, 2009; Paradies et al. 2013). More recent fecal examination methods such as the sensitive flotation method FLOTAC (Schnyder et al. 2010b) might improve performance. Fecal PCR appeared to give more consistent results than larval counts in the present study, but some negative results were nevertheless obtained despite the proven presence of adult worms. Fecal PCR was also shown to give variable results in natural infections (Jefferies et al. 2009).

In conclusion, the present study evaluated an array of potential diagnostic tests in a group of 
dogs experimentally infected for the purpose of a trial of anthelmintic efficacy. Tests varied in the timing and consistency of positive results. Circulating parasite antigen and specific antibodies in serological samples appeared early in the course of infection and remained persistently above the positive cut-off throughout patency, before declining in treated dogs over the few weeks following administration of anthelmintic. Other tests gave more variable results over the patent period. It is likely that in clinical practice, different tests will have diagnostic value in various cases, alone or in combination (Jefferies et al. 2011; Schnyder et al. 2014). In respect thereof, the recent commercial availability of a rapid in-clinic assay (Angio Detect $^{\mathrm{TM}}$ Test, IDEXX Laboratories, Westbrook, Maine, USA) for the serological detection of circulating antigen showed a good sensitivity and a very high specificity (Schnyder et al. 2014), and may represent a valid alternative to be broadly applied in veterinary practices in dogs with suspected canine angiostrongylosis. With this test, earliest positive results were obtained starting from 9 wpi, while after anthelmintic treatment seropositive dogs turned negative again within 3-7 weeks after treatment. The direct comparison with the ELISA detecting circulating antigen showed a delay of approximately 3-4 weeks for antigen detection by the Angio Detect ${ }^{\mathrm{TM}}$ Test. Contemporaneously, the utilization of broadly applied ELISAs in epidemiological surveys can improve the evidence base available to practitioners, especially concerning local risk of disease (Guardone et al. 2013; Schnyder et al. $2013 a, b)$.

\section{ACKNOWLEDGEMENTS}

The authors sincerely thank Dr med. vet. Barbara Riond from the Clinical Laboratory for the sera of blood donor dogs as well as of further randomly selected samples and acknowledge Isabelle Tanner and Katja Huggel for their contribution in the production of monoclonal antibodies and Lucia Kohler for intense laboratory tasks.

\section{FINANCIAL SUPPORT}

M. Schnyder acknowledges Bayer Animal Health $\mathrm{GmbH}$, Germany, for the financial contribution.

\section{REFERENCES}

Al-Sabi, M. N., Deplazes, P., Webster, P., Willesen, J. L., Davidson, R. K. and Kapel, C. M. (2010). PCR detection of Angiostrongylus vasorum in faecal samples of dogs and foxes. Parasitology Research 107, 135-140.

Barutzki, D. and Schaper, R. (2009). Natural infections of Angiostrongylus vasorum and Crenosoma vulpis in dogs in Germany (2007-2009). Parasitology Research 105(Suppl. 1), 39-48.

Conboy, G. (2011). Canine angiostrongylosis: the French heartworm: an emerging threat in North America. Veterinary Parasitology 176, 382-389. Cury, M. C. and Lima, W.S. (1996). Rupture of femoral artery in a dog infected with Angiostrongylus vasorum. Veterinary Parasitology 65, 313-315.
Deplazes, P., Eckert, J., v. Samson-Himmelstjerna, G. and Zahner, H. (2013). Lehrbuch der Parasitologie für die Tiermedizin. Enke Verlag, Stuttgart. Ferdushy, T., Kape1, C.M., Webster, P., Al-Sabi, M. N. and Gronvold, J. (2009). The occurrence of Angiostrongylus vasorum in terrestrial slugs from forests and parks in the Copenhagen area, Denmark. Fournal of Helminthology 83, 379-383.

Guardone, L., Schnyder, M., Macchioni, F., Deplazes, P. and Magi, M. (2013). Serological detection of circulating Angiostrongylus vasorum antigen and specific antibodies in dogs from central and northern Italy. Veterinary Parasitology 192, 192-198.

Guilhon, J. (1963). Recherches sur le cycle évolutif du Strongle des vaisseaux du chien. Bulletin de l'Académie Vétérinaire 36, 431-442.

Humm, K. and Adamantos, S. (2010). Is evaluation of a faecal smear a useful technique in the diagnosis of canine pulmonary angiostrongylosis? Fournal of Small Animal Practice 51, 200-203.

Jefferies, R., Morgan, E. R. and Shaw, S. E. (2009). A SYBR green realtime PCR assay for the detection of the nematode Angiostrongylus vasorum in definitive and intermediate hosts. Veterinary Parasitology 166, 112-118. Jefferies, R., Morgan, E. R., Helm, J., Robinson, M. and Shaw, S. E. (2011). Improved detection of canine Angiostrongylus vasorum infection using real-time PCR and indirect ELISA. Parasitology Research 109, $1577-1583$.

King, M. C. A., Grose, R. M. R. and Startup, G. (1994). Angiostrongylus vasorum in the anterior chamber of a dog's eye. Fournal of Small Animal Practice 35, 326-328.

Kistler, W. M., Brown, J.D., Allison, A. B., Nemeth, N. M. and Yabsley, M. J. (2014). First report of Angiostrongylus vasorum and Hepatozoon from a red fox (Vulpes vulpes) from West Virginia, USA. Veterinay Parasitology 200, 216-220.

Koch, J. and Willesen, J. L. (2009). Canine pulmonary angiostrongylosis: an update. Veterinary fournal 179, 348-359.

Matos, J. M., Schnyder, M., Bektas, R., Makara, M., Kutter, A., Jenni, S., Deplazes, P. and Glaus, T. (2012). Recruitment of arteriovenous pulmonary shunts may attenuate the development of pulmonary hypertension in dogs experimentally infected with Angiostrongylus vasorum. Fournal of Veterinary Cardiology 14, 313-322.

McGarry, J. W. and Morgan, E. R. (2009). Identification of first-stage larvae of metastrongyles from dogs. Veterinary Record 165, 258-261.

Morgan, E. and Shaw, S. (2010). Angiostrongylus vasorum infection in dogs: continuing spread and developments in diagnosis and treatment. Fournal of Small Animal Practice 51, 616-621.

Oliveira-Junior, S. D., Barcante, J. M., Barcante, T. A., Ribeiro, V. M. and Lima, W. S. (2004). Ectopic location of adult worms and first-stage larvae of Angiostrongylus vasorum in an infected dog. Veterinary Parasitology 121, 293-296.

Oliveira-Junior, S. D., Barcante, J. M., Barcante, T. A., Dias, S. R. and Lima, W.S. (2006). Larval output of infected and re-infected dogs with Angiostrongylus vasorum (Baillet, 1866) Kamensky, 1905. Veterinary Parasitology 141, 101-106.

Paradies, P., Schnyder, M., Capogna, A., Lia, R. P. and Sasanelli, M. (2013). Canine angiostrongylosis in naturally infected dogs: clinical approach and monitoring of infection after treatment. Scientific World fournal 2013, 702056.

Schnyder, M., Fahrion, A., Ossent, P., Kohler, L., Webster, P., Heine, J. and Deplazes, P. (2009). Larvicidal effect of imidacloprid/moxidectin spot-on solution in dogs experimentally inoculated with Angiostrongylus vasorum. Veterinary Parasitolology 166, 326-332.

Schnyder, M., Fahrion, A., Riond, B., Ossent, P., Webster, P., Kranjc, A., Glaus, T. and Deplazes, P. (2010a). Clinical, laboratory and pathological findings in dogs experimentally infected with Angiostrongylus vasorum. Parasitology Research 107, 1471-1480.

Schnyder, M., Maurelli, M.P., Morgoglione, M. E., Kohler, L. Deplazes, P., Torgerson, P., Cringoli, G. and Rinaldi, L. (2010b). Comparison of faecal techniques including FLOTAC for copromicroscopic detection of first stage larvae of Angiostrongylus vasorum. Parasitology Research 109, 63-69.

Schnyder, M., Tanner, I., Webster, P., Barutzki, D. and Deplazes, P. (2011). An ELISA for sensitive and specific detection of circulating antigen of Angiostrongylus vasorum in serum samples of naturally and experimentally infected dogs. Veterinary Parasitology 179, 152-158.

Schnyder, M., Schaper, R., Bilbrough, G., Morgan, E. R. and Deplazes, P. (2013a). Seroepidemiological survey for canine angiostrongylosis in dogs from Germany and the UK using combined detection of Angiostrongylus vasorum antigen and specific antibodies. Parasitology 140, 1442-1450.

Schnyder, M., Schaper, R., Pantchev, N., Kowalska, D. Szwedko, A. and Deplazes, P. (2013b). Serological detection of 
circulating Angiostrongylus vasorum antigen- and parasite-specific antibodies in dogs from Poland. Parasitology Research 112(Suppl. 1), 109-117. Schnyder, M., Stebler, K., Naucke, T. J., Lorentz, S. and Deplazes, P. (2014). Evaluation of a rapid device for serological in-clinic diagnosis of canine angiostrongylosis. Parasites and Vectors 7, 72.

Schucan, A., Schnyder, M., Tanner, I., Barutzki, D., Traversa, D. and Deplazes, P. (2012). Detection of specific antibodies in dogs infected with Angiostrongylus vasorum. Veterinary Parasitology 185, 216-224.
Staebler, S., Ochs, H., Steffen, F., Naegeli, F., Borel, N., SieberRuckstuhl, N. and Deplazes, P. (2005). Autochthonous infections with Angiostrongylus vasorum in dogs in Switzerland and Germany (in German). Schweizer Archiv fur Tierheilkunde 147, 121-127.

Traversa, D., Iorio, R. and Otranto, D. (2008). Diagnostic and clinical implications of a nested PCR specific for ribosomal DNA of the feline lungworm Aelurostrongylus abstrusus (Nematoda, Strongylida). Fournal of Clinical Microbiology 46, 1811-1817. 